\title{
A Preliminary Inventory of the Transformations of Scientific Imaging
}

\author{
Robert Rosenberger \\ Georgia Institute of Technology \\ School of Public Policy \\ 685 Cherry St., DM Smith Building \\ Atlanta, GA 31201, USA \\ Email: robert.rosenberger@pubpolicy.gatech.edu
}

\begin{abstract}
Imaging technologies "transform" an object of study into something we can visually perceive in the form of an image. In science and medicine, imaging technologies enact a large variety of transformations, sometimes changing the spatiality of an object of study (e.g., making a small thing big enough to see, bringing close something far away, etc.), or changing its temporality (e.g., providing a picture of a single moment). I make use of the postphenomenological philosophical perspective, and in particular the work of its founder, Don Ihde, for guidance in exploring the different ways that imaging technologies transform our world in the process of rendering it available to visual perception. The main project of this paper is to develop a provisional categorization of a large variety of image transformations common to science and medicine.
\end{abstract}

Keywords: mediation, medical imaging, philosophy of technology, postphenomenology, scientific imaging

\section{Introduction}

A picture of a thing in the world is of course not the same as that thing itself. The content of that picture is something different than the actual thing of which that picture has been taken. For example, the picture is flat. The thing may not be. The picture has borders. The thing was out in the endless world. We could enumerate many differences, many ways in which the camera has transformed that thing out in the world into the form of an image. 
The kinds of transformations that are made by imaging technologies are crucial to the practice of science and medicine. Sometimes these transformations are what make possible a particular line of scientific study or medical investigation in the first place. Here, I offer a brainstorm of these various transformations. When imaging technologies - from cameras to electron microscopes to fMRI scansproduce images, what kinds of transformations do they enact? Since imaging technologies are crucial parts of scientific research and medical diagnoses, it is important to fully consider the categories of differences that exist between our objects of study on the one hand, and the images we make of those objects on the other.

To do so, I make use of the postphenomenological philosophical perspective. This school of thought specializes in the deep description of how users experience technologies, and there is a tradition of work within this school focused on scientific and medical imaging technologies. Building on this work, I develop a preliminary list of the transformations that imaging technologies enact.

\section{Experience and images}

The topic of scientific imaging is complex, involving questions of epistemology, design, scientific practice, methodology, and even ethics and politics. One essential element is an understanding of how human users experience images.

The "postphenomenological" school of thought is useful for drawing out these kinds of issues. This perspective integrates ideas from the history of phenomenology with some of the philosophical commitments of American pragmatism to explore the concrete details of human-technology relations (e.g., Ihde, 2009; Verbeek, 2011; Rosenberger \& Verbeek, 2015; Aagaard et al., 2018; Hasse, 2020). The framework of concepts coming out of postphenomenology has proven especially useful for the study of the experience of imaging. Postphenomenological studies have been conducted on imaging in a variety of contexts, including physics laboratories, neurobiological sample freezing, cytodiagnosis, the climate history of Mars, radiology, non-invasive brain stimulation, as well as a variety of investigations into imaging in everyday contexts, from computing, to smartphone apps, to wearable tech (e.g., Hasse, 2008; Rosenberger, 2011; 2013; Forss, 2012; Friis, 2017; Secomandi, 2017; Kudina \& Verbeek, 2019; de Boer et al.; Fried, forthcoming). These studies are often interdisciplinary, bringing 
together methods from multiple disciplines, including philosophy, sociology, psychology, ethnomethodology, and media studies.

All of this work on the postphenomenological study of imaging builds on the foundational insights offered by Don Ihde. Ihde is the founding figure of postphenomenology, and he has maintained a career-long fascination with the topic of imaging in science. The essential work in this line of research is Ihde's 1998 book, Expanding Hermeneutics: Visualism in Science, and in particular the final, fourth part of that book which he refers to as a "minimonograph" on the topic of scientific imaging. Recently, I have developed a sort of sequel to that work, a minimonograph of my own entitled 'A Primer on Postphenomenology and Image Reading,' in the forthcoming book Postphenomenology and Imaging: How to Read Technology, edited by me and Samantha Jo Fried. In what follows, I continue this line of inquiry by focusing specifically on the topic of the technological transformations of imaging technologies.

Imaging is crucial to the practice of science and medicine because often these technologies are used to make it possible to visually perceive something that would be impossible otherwise. Telescopes enable us to see things that are farther away than we could possibly travel. X-rays enable us to see inside a living human body without having to open it up. And so on. In postphenomenological terms, a technology can be conceived as a form of "mediation," that is, as something that comes between the human user and the world and that changes and reshapes that relationship. Imaging technologies are a form of technological mediation, one that "transforms" the world into a specific form that is available to human visual perception: an image. And, like all forms of technological mediation, the mediation of imaging technologies is neither neutral nor innocent.

In exploring the phenomenology of imaging, there are many things to consider. There is the gestalt quality of the apprehension of images; for someone already familiar with a kind of image, much of an image's meaning will emerge in a perceptual gestalt (e.g., Ihde, 1998, pp. 161-162). There is also the multistability of images; images can possess more than only one meaning, and can be perceived and interpreted in more than only one way (e.g., Rosenberger, forthcoming, part 2). Images may fit into multiple social settings, taking on different meanings in different communities. Images are subject to issues of cultural learning, taking on meaning in relation to local background knowledge. Imaging technologies are material objects, and as such imply trajectories for development and for research. The topic of scientific imaging technologies, of course, also has implications for the epistemology of science. And on and on. 
Here I want to focus on one issue in particular: the transformations of imaging technologies. Ihde writes that, "the 'reduction' of perception to a monodimension - the visual — is already an abstraction from the lived experience of active perception within a world" (Ihde, 1998, p. 161). Images are largely mono-sensory technologies, engaging human visual perception. This stands in stark contrast to the multisensory lived body within the world.

In addition to this basic change from a full-bodied sensorial engagement with an object of study to a merely visual one, what we could call a "mono-sensorial transformation", I hope to catalog here a large assortment of additional transformations enacted by imaging technologies. Let us continue to take our cue from Ihde's writing. In his extensive reflections on Galileo, he writes, "If the dramatic appearance of relative distance (space) was the forefront fascination with Galileo's telescope, one might by contrast note that it is the dramatic appearance of a transformation of time which photography brought to scientific attention" (Ihde, 1998, p. 164). These two transformations-the transformation to the spatiality of an object of study, and to its temporalitycan serve as two of our main categories, categories that below I will refine into a catalog of further subcategories of spatial and temporal transformations. As a third main category, let us draw on Ihde's distinction between 'isomorphic' and 'constructed' images, that is, images that bear a correspondence in shape to the object of study, and those that involve differences generated to visualize things (such as the addition of "false color"). In the next section, I take an exploratory pass at listing a variety of categories of transformations enacted by imaging technologies.

This attempt at generating a list of transformation types constitutes the body of this paper.

\section{A provisional enumeration of image transformations}

In what follows in this section, I attempt to develop a preliminary inventory of the ways that imaging technologies transform an object of study in the process of rendering it into the form of an image. I refer to this as a "preliminary" inventory to indicate its provisional and exploratory nature. This proposed set of concepts is surely not comprehensive. And while I propose an abundance of "titles" for these categories of transformations here, the spirit of these propositions is exploratory. 
With this initial brainstorm, I am hoping to put this topic of articulating the categories of imaging transformations on the table for postphenomenological research. I propose three main categories—spatial, temporal, and constructional transformations - which will then be further refined.

\section{SPATIAL TRANSFORMATIONS}

The first main category of transformations to consider here is what we can call 'spatial transformations,' i.e., changes to the spatiality of our object of study through the actions of imaging technologies. Within these, let us include all manner of changes in the size and shape of the object of study, the location of our perspective upon that object, and the distance between the user and the object under study.

\section{Distantial transformations}

One common form of transformation is that of distance, what we could call a 'distantial transformation.' Imaging technologies may change the distantial relationship between the viewer and an object of study. That is, they change-at least in an experiential sense-the quantity of space that separates an object of study and the viewer. It seems that most often the distantial transformation sought is one in which a faraway object of study is brought near. As Ihde puts it, "what was previously more 'distant' is now 'closer" (Ihde, 1998, p. 154). In the case of some potential objects of study, distantial transformations may change something otherwise impossible to see because it is too far away into a visually perceptual form.

For example, imagine a picture of a hummingbird taken with a camera equipped with a powerful zoom lens. The photographer may be far away from the bird in terms of measurable distance, but the transformative technological mediation of the lens brings the animal experientially close. This is true as well for the picture taken of the bird; among the many transformations rendered to the object of study by the camera in changing it from a living and moving being into a static picture, one of those changes includes the distance between the viewer and the hummingbird. Although in a reduced and greatly altered form, the bird is locally present within the picture to the viewer.

Of course, these kinds of distantial transformations can be crucial to scientific research, such as in the cases of telescopic and satellite imaging. Spacecraft, such as Cassini, transform the distance between us and the outer planets of our solar 
system, enabling visual apprehension of an altered version of these objects of study in the form of images.

\section{Voluminal transformations}

With the notion of 'voluminal transformations,' I refer to changes to size. As Ihde observes,

The trajectory into the microscopic, of course, explodes in the nineteenth and twentieth centuries, with electron microscopes, scanning, tunneling processes, and on to the processes which even produce images of atoms and atom surface structure (Ihde, 1998, p. 166).

As is the case for all of these transformations, the meaning of image content may not be obvious to a viewer who is not already familiar with its context. Coherent and meaningful visual apprehension of image content may even require extensive training. This can be the case for some of the paradigmatic examples of imaging research in science and medicine that center on voluminal transformations. For example, think of electron microscope images of parts of cells. Such objects of study cannot be perceived with normal unaided human vision. These kinds of laboratory imaging technologies, and their associated imaging processes (such as sample preparation techniques), render these objects of study available to human visual perception in the first place. And seeing such an object of study for what it is-cells, rather than incoherent structures and blurs-calls for training in image reading.

In order for a meaningful human-technology relation to happen in this case, the object of study must be transformed by the imaging technology, and the viewer's visual perception must be readied as well through training and accustomization. I have been developing the notion of 'hermeneutic strategies' to help emphasize and articulate this human side of human-technology relations to images, and to point to the trained perceptual interpretive work a viewer brings to the act of perceiving an image in one way rather than another (e.g., Rosenberger, forthcoming).

\section{Dimensional transformations}

A change to the spatial depth of the object of study can be referred to as a 'dimensional transformation.' The most common dimensional transformation rendered by imaging technologies is a change from the full three-dimensionality of an object of study to a two-dimensional image. Images are, of course, often flat, reduced to two dimensions across a page or screen. 
To adjust for these changes, special alterations are sometimes made to images to convey the lost depth, such as shadowing effects or false color. Even images with $3 \mathrm{D}$ content are often themselves $2 \mathrm{D}$ images, and can require a kind of hermeneutic training in order to understand the content being conveyed.

It is possible, too, that if the image is itself three-dimensional, such as in the case of holography, there still could be transformations made to the dimensionality of the object of study.

\section{Morphological transformations}

We can use the term 'morphological transformations' to refer to changes in shape. Through the imaging process, an object of study may be be altered in terms of its morphology in its transition from 'object' to 'image of that object.'

One common example is the warping of the shapes of countries in mapmaking. In the transformation from the curved earth to the flat, two-dimensional map, not only is there a dimensional transformation, the actual shapes of the countries are changed as well. Or, take the example of fMRI brain scans. Brain data may at times be mapped to a standardized spatial atlas system, such as Talairach coordinates, to make it easier to compare samples from different subjects. And there is surely some conceptual bleed here with the notion of dimensional transformations above; the morphology of image content (e.g., the height of mountain ranges, the curve of rounded surfaces) might be obscured within the content of an image as an effect of a dimensional change from the full dimensions of the world to a $2 \mathrm{D}$ image.

\section{Compositional transformations}

The creation of an image of an object of study involves the removal of that object from its context within the larger world. Ihde writes,

in what may seem too obvious and trivial, whether one is referring to MTV or an MRI scan of the brain, the image is framed space. This implies what is presented is presented as already from distinct ordinary or lived-body space precisely by the limited and selected-out framing of the image presentation. (Ihde, 1998, p. 91)

We could use the term 'compositional transformations' to refer to the changes relevant to an image's status as a selection of a spatial area. 
This notion puts a spotlight on an often under-acknowledged and yet everpresent aspect of images: their borders. Images are cut off "at the sides." There are boundaries that comprise the edges of the image. And as such, images constitute a form of curation. What is contained within the borders, rather than lost off the edge of the image, is at least implicitly presented as the proper object of attention.

There are interesting phenomenological and hermeneutic elements of the user experience of these transformations. Often, for trained users accustomed to perceiving a particular kind of image, the borders of a particular image are not experienced as the ending of image content. The content within the image is experienced as at least implicitly continuing on past those borders.

\section{Positional transformations}

We can use the term 'positional transformations' to refer to changes in the perspective from which the content of the image is viewed. As Ihde puts it, "Reflexively, the embodied 'here' of the observer not only may be noted but is a constant to all sensory perspectivalism" (Ihde, 1998, p. 171). And this is no less true for the creation and use of images. If we return to the example of the electron microscope image of the cell, we could consider whether the implied positionality of image viewership is outside the cell looking upon it, or instead inside the cell and viewing the interior (such as the interior surface of the cell wall). Or, return to the example of Cassini images of Saturn, taken from the vantage point of a position that puts the viewer implicitly suspended within the planet's orbit.

Positional transformations can include a variety of kinds of changes that can be enumerated as subcategories. This could help to bring some further refinement to our articulation of the various positional transformations at issue in imaging work. Let us consider a few of the possible transformations to viewership location, sight angle, point outside or inside an object of analysis, and such.

We could use the term 'locational transformation' to refer to changes in the understood spatial location of the point of viewership. If we consider examples of nature photography, the implied location of the viewer is set in the context of the scene of the image (albeit in an experientially reduced sense). For example, an image of a leopard lounging in a tree does not merely represent a distantial transformation of the space between our location on the planet and that of the leopard's tree; we are implicitly put in the position of standing in the desert beside the tree. Perhaps our position is on the ground looking up at the tree 
branch above our heads. Or perhaps it is instead implicitly one in which we are floating in the air in front of the leopard. Return again to the example of an orbiter spacecraft. If our satellite spacecraft provides us with an image of another planet, then not only is the distance between us and that planet transformed (in this case, reduced), the location of our vantage point has been transformed to one in which we are suspended in space. That spot in space is one for which we would require technological assistance (to be alive there, and also to maintain that altitude) were we to actually be present.

We could contrast this with what we could call an 'orientational transformation.' This could refer to a change in our angle of vision upon an object of study. If the notion of locational transformations addresses the question of "from what spot?" then the notion of orientational transformations addresses the question of "from what vantage point?" For example, we could contrast the differing perspectives upon the surface of Mars afforded by an orbiting satellite craft and a rover. Both enact a distantial transformation, bringing the faraway Martian surface to us here on earth. And both enact a slightly different locational transformation, providing a perspective of the planet from a spot as if we are either hovering above it, or instead driving along the surface. These different locational transformations involve a corresponding difference in orientational transformation: the satellite orbiter provides a bird's-eye view, and the rover provides a view from on the ground. Or for example, we could consider the different orientational transformations involved in fMRI brain imaging. Such images appear to most often display a bird's-eye view, as if one is looking down into someone's head from a vantage point just above that brain. However, of course fMRI images are taken from different angles as well, for example, sometimes offering a side-view profile.

We could also consider what could be called "locationally dynamical transformations," i.e., transformations to the mobility of our relationship to the imaged space. Return once again to the example of the photograph of the hummingbird. As a photo, the image is a fixed one. This fixed character is not only temporal (which I will get to below), but also spatial. In the case of an object of study like a hummingbird, were we to be there present with it, we could take up different spatial positions while looking at it (say, walking left or right, or toward or away from it). However in this example, in the process through which our object of study is changed into an image, there is a transformation from the spatially dynamic character of the world, to the space of the image in which that spatial location has become fixed. Of course there are also other possibilities further than merely fully dynamic or entirely fixed. 
We could use the term 'ingressional transformation' to refer to a change in perspective afforded by an imaging technology that enables the viewer to peer inside an object of study. Ihde writes,

today's MRI scan, CT tomography, PET scans, and sonograms all are variants upon the depiction of interiorities. Each of these processes not only does its depicting by different means but also produces different visual selectivities which vary what is more or less transparent and what is more or less complete (Ihde, 1998, p. 165).

These transformations to our ability to acquire visual access to the interiority of an object of study can, of course, be crucial to medical diagnoses and scientific investigations. They often involve an ability to visualize something that would be impossible to see in the same way even if we were there present with the object of study (and also had the ability to crack it open). To continue with the fMRI brain imaging example, it is indeed possible to look at a human brain in an opened scull, and brain surgeries involve looking at a brain of someone who continues to be alive. However, the fMRI provides a view of a working brain's real-time changes in blood oxygenation (and thus, brain activity), a "selectivity" we could not see without the power of these technological transformations.

\section{Resolutional transformations}

Images of an object of study come in greater and lesser levels of resolution, often engendering a change that is crucial to an investigation. We could use the term 'resolutional transformations' to refer to the ways in which image resolution varies in our studies. Perhaps a satellite image of the Earth's surface taken from space involves several positional transformations (locational, orientational) that enable us to see the landscapes of our planet in ways that we could never otherwise perceive visually in an unaided manner. Some examples of these kinds of satellite images come in the form of a static picture, and one with limited resolution. In such an instance of resolutional transformation, perhaps the tradeoff includes the ability to see the landscape from the satellite's perspective, but with a limited resolution in which houses appear as small and blurry boxes, etc. There are, of course, examples in which the image resolution is such that a user can zoom in more and more, again in a way that involves technological transformations significantly different from unaided human bodily perception. 


\section{TEMPORAL TRANSFORMATIONS}

A second main category of transformations is what we can refer to as 'temporal transformations,' i.e., changes to the temporality of our object of study through the actions of imaging technologies. Within these, let us include all manner of changes in temporal fixity, duration, speed, as well as the directionality of the temporal flow captured within our images.

\section{Temporally dynamical transformations}

A common and significant change to an object of study rendered by imaging technologies is the transformation from its place in the advancing flow of time into an image of just a single fixed moment. Ihde observes, "The photograph 'stops time,' and the technological trajectory implicitly suggested within it is the ever more precise micro-instant that can be captured" (Ihde, 1998, p. 164). This kind of transformation is critical to our example of the photo of the hummingbird. Of course, one of the things that makes hummingbirds such interesting creatures is that their wings move faster than what is perceptible to the human eye. However, by capturing an instant of their fluttering, a picture makes it possible to see the hummingbird's wings even while it is flying, but in a transformed manner: motionless midflight.

Temporally dynamical process-to-moment transformations rendered by imaging technologies can be found across science and medicine. For example, ultrasound imaging centrally enacts an ingressional transformation, providing a view inside the human body in real time. And yet, while enabling real time views, snapshots of moments of ultrasound scans are often taken so that individual moments of the imaging process (such as when a good angle on the object of study is achieved) can be observed more closely in detail later. Transformations to temporal dynamics are, of course, at times crucial to research. For example, neurotransmission - the ejection of neurotransmitter from inside of a neuron terminal out into the synapse-is a biological process that occurs in the order of milliseconds. Therefore, imaging research on neurotransmission must not only enact ingressional and voluminal transformations to neurons in order to respectively remove them from inside the body and enlarge them. They must also transform a process that occurs at a speed otherwise too fast to see into a form open to human visual perception: a fixed image.

As with all of the transformations at issue in this article, this transformation to temporal fixity is sometimes the exact goal of the imaging process. And sometimes 
it is, instead, a side effect, an instrumental artifact. For example, perhaps the goal in taking a picture of the hummingbird is exactly to freeze it in flight; as something too fast to see, the picture transforms the hummingbird's wings into something possible to visualize. Or, for example, perhaps the goal is not specifically to capture one moment of the hummingbird's flight, but something else related to that. Imagine that our goal is to follow the hummingbird around and observe its behaviors. As something that zips around quickly, we snap a bunch of pictures of the hummingbird in flight as it whizzes from flower to flower. This temporally dynamical transformation makes it possible to observe and study at leisure several moments of the otherwise quick and itinerant bird. But researchers might sometimes look at one image and wish that it was possible to observe a moment that had occurred just after or just before a particular picture was taken, the fixity a side effect of photography.

Of course, other transformations of this temporally dynamical sort are possible. Instead of the transformation from process to moment as discussed so far, we can also include the creation of video clips, segmented temporal spans that capture a length of time.

\section{Velocital transformations}

We could use the term 'velocital transformations' to refer to changes rendered by an imaging technology to the speed of an object of study. We could go further and use the term 'accelerational transformation' to refer to changes that increase the speed, and we could use the term 'decelerational transformation' to refer to changes that decrease it.

For example, we could imagine scientists who are observing children performing tasks in a laboratory setting, looking to observe and quantify specific behaviors. To perform this study, they make use of an imaging technology, a video camera that can record the children for extended periods. Searching for particular behaviors performed by the children onscreen, these researchers may watch the recording on "fast forward," speeding up the tape and scanning for particular things. Perhaps when a targeted behavior is noticed, the researchers then reduce the speed of the tape to a pace slower than real life to better catalog the details of that behavior. It is like when referees use "slow-motion instant replay" video to re-watch a sports play at reduced speed to observe details too fast to see otherwise. 


\section{Instantial transformations}

Imaging technologies often pull an object of study out of the present instant. We could use the term 'instantial transformation' to refer to the change in tense rendered by imaging processes. Many of the examples discussed so far-from the picture of the hummingbird, to the image of a moment of neurotransmission, to the video recording of the child development laboratory-involve instantial transformations. They do not provide a view of their object of study at the present moment; they provide a view of a previous moment, capturing a moment of time that took place in the past.

A distinction can be drawn between whether instantial transformations provide a vantage point upon instants from the past or those from the future. We could use the term 'post-spectival transformation' for transformations that change our perspective from the present into one that enables us to visualize something that has already happened. When the referees look to the slow-motion instant replay in sports, in addition to the decelerational transformation, they also make use of a post-spectival one; they want to see an event that has already occurred. Compare this with, say, the imaged readouts of computer simulations of weather forecasts. Such images purport to display what could be a 'pre-spectival transformation', making visible information about the future.

\section{Chronological transformations}

We could use the term 'chronological transformation' to refer to changes in temporal sequence. Sometimes our imaging technology can be used to transform the order in which time passes regarding our object of study. Perhaps the most straightforward example would be a video that reverses the temporality of a sequence of events, enabling us to watch something occur backwards. But there are other rearrangements of sequence possible. For example, think of static images that are made to display multiple events at once, even though those events did not happen at the same time, such as a picture that presents multiple phases of the moon beside one another.

\section{CONSTRUCTIONAL TRANSFORMATIONS}

Ihde has offered a useful distinction between images whose content bears at least some structural resemblance to the object of study, and those that do not. He writes, "Within the multiple uses of scientific imaging, there is a spectrum which does run from partial isomorphism to variations upon isomorphism 
which vary away from the 'literal' or copy form, toward a certain kind of 'fictive' or technologically enhanced form of variation" (Ihde, 1998, p. 92). In Ihde's terminology, images are "isomorphic" if their content shares some structural correspondence with the thing in the world being imaged. Many of the examples considered so far maintain an isomorphic resemblance with the object of study, including the fMRI brain scan, Mars satellite pictures, video instant replay, and hummingbird photographs. We can compare this to "non-isomorphic" images, in which imaging technologies provide a readout available to our visual perception, but one for which there is no temporal and spatial relationship with the object of study. One example is the spectral line readouts that are used to identify molecular composition. Another example are the "charts, graphs, models, and the whole range of 'readable' inscriptions which remain visual, but which are no longer isomorphic with the referent objects or 'things themselves"' (Ihde, 1998, p. 167).

This raises another issue: images are often altered in order to convey information. Isomorphic and non-isomorphic images may be changed through additional imaging processes that add further information in this visual form. Ihde refers to these changes as image 'constructions.' Following the terminology developed here, we could refer to these as 'constructional transformations.' A paradigmatic example is false color enhancements added to an image to convey information. False colors are added to images of everything from geographic maps to convey mineral deposits, to fMRI brain scans to convey activity levels. Other straightforward examples are words, arrows, boxes, and icons added to images, such as those used on meteorological maps. There is some conceptual bleed here between the kinds of transformations we have identified above, and what we might want to call constructional. But other examples could include the use of imaging technologies to enable viewers to visualize phenomena otherwise impossible, such as night vision, heat vision, or the enhancement of images of celestial bodies to include light spectra beyond what can be seen by the naked eye.

It seems to me that this third category is ripe for further conceptual refinement. 


\section{Discussion}

It is important to note again the provisional spirit of the above list of image transformations. I offer these thoughts as a kind of brainstorm. The use of images is, of course, pervasive across the various fields of science and medicine. For those familiar with different imaging practices and examples than the ones I have studied in my own empirical work, further or different categories of image transformations may be obvious. The point here is to put this topic on the table. I hope to have shown that the basic concepts of mediation and transformation are useful starting points for this kind of work. It is certainly true as well that a number of further notions from postphenomenology, the philosophy of science, and STS accounts of imaging practice would also be useful in describing the imaging practices reviewed above. (By leaning almost exclusively on 'transformation' terminology, I have been trying to keep things somewhat simpler in terms of theory, and to focus on the identification of these different concepts.)

Ihde's designation of spatial and temporal transformations is a useful distinction, and so are his reflections of the isomorphic and constructed qualities of images. However, I suggest that we should see these notions as starting points. There appears to me to be room to run in terms of expanding and refining this conceptual framework. And I hope to have proven that general point through the above brainstorming session.

It is my contention that this kind of conceptual refinement of the variety of transformations enacted by our imaging technologies can be of practical value to scientific and medical practice. In my observation, disagreements over image interpretation often occur over these exact issues. The kinds of transformations listed above are not incidental to medical and scientific imaging: by making something visible that was otherwise imperceptible, they are exactly what makes medical diagnoses and scientific discoveries possible. But in the process of making those things possible, they change the object of study. And it is within these changes that disagreements can occur. Sometimes they are couched as debates about the nature of the object of study itself. And yet, much of the substance of the disagreement may be (more or less implicitly) about the implications of image transformations-whether they are the transformations that have made something possible to see, or those that are "side-effect" artifacts of imaging processes. 
In this way, in addition to contributing to our theoretical understanding of scientific medical imaging practice, investigations of imaging transformations could be of potential use to the practice of medicine and science.

\section{References}

Aagaard, J.; Friis, J. K. B.; Sorenson, J.; Tafdrup, O. \& Hasse, C., eds. (2018), Postphenomenological Methodologies: New Ways in Mediating Techno-Human Relationships, Lanham: Lexington Books.

de Boer, B.; te Molder, H. \& Verbeek, P.-P. (forthcoming), 'Constituting 'visual attention': On the mediating role of brain stimulation and brain imaging technologies in neuroscientific practice,' Science as Culture.

Forss, A. (2012), 'Cells and the (imaginary) patient: The multistable practitionertechnology-cell interface in the cytology laboratory,' Medicine, Health Care \& Philosophy, no. 15, pp. 295-308. https://doi.org/10.1007/s11019-011-9325-0

Fried, S. J. (forthcoming), 'Satellites, war, climate change, and the environment: Are we at risk for environmental deskilling?' AI \& Society. https://doi.org/10.1007/s00146-020-01047-2

Friis, J. K. B. O. (2017), 'Gestalt descriptions, embodiments, and medical image interpretation,' AI \& Society, vol. 32, pp. 209-218. https://doi.org/10.1007/s00146-015-0615-6

Hasse, C. (2008), 'Postphenomenology: Learning cultural perception in science,' Human Studies, vol. 31, no. 1, pp. 43-61. https://doi.org/10.1007/s10746-007-9075-4

Hasse, C. (2020), Posthumanist Learning: What Robots and Cyborgs Teach Us About Being Ultra-Social, London: Routledge. https://doi.org/10.4324/9781315647661

Ihde, D. (1998), Expanding Hermeneutics: Visualism in Science, Evanston: Northwestern University Press.

Ihde, D. (2009), Postphenomenology and Technoscience: The Peking University Lectures, Albany: SUNY Press.

Kudina, O. \& Verbeek, P.-P. (2019), 'Ethics from within: Google Glass, the Collingridge Dilemma, and the mediated value of privacy,' Science, Technology \& Human Values, vol. 44, no. 2, pp. 291-314. https://doi.org/10.1177/0162243918793711

Rosenberger, R. (2011), 'A case study in the applied philosophy of imaging: The synaptic vesicle debate,' Science, Technology, \& Human Values, vol. 36, no. 1, pp. 6-32. https://doi.org/10.1177/0162243909337117

Rosenberger, R. (2013), 'Mediating Mars: Perceptual experience and scientific imaging technologies,' Foundations of Science, vol. 18, no. 75-91. https://doi.org/10.1007/s10699-012-9286-7 
A Preliminary Inventory of the Transformations of Scientific Imaging

Rosenberger, R. (forthcoming), 'A primer on postphenomenology and image reading,' in S. J. Fried \& R. Rosenberger (eds.) Postphenomenology and Imaging: How to Read Technology, Lanham: Lexington Books.

Rosenberger, R. \& Verbeek, P.-P., eds. (2015), Postphenomenological Investigations: Essays on Human-Technology Relations, Lanham: Lexington Books.

Secomandi, F. (2017), 'Digital images and multistability in design practice,' in Y. Van Den Eede, S. Irwin \& G. Wellner (eds.) Postphenomenology and Media: Essays on Human-Media-World Relations, Lanham: Lexington Books, pp. 123-143.

Verbeek, P.-P. (2011), Moralizing Technology: Understanding and Designing the Morality of Things, Chicago: Chicago University Press.

https://doi.org/10.7208/chicago/9780226852904.001.0001

Robert Rosenberger works in the School of Public Policy at the Georgia Institute of Technology. His mini-monograph is entitled Callous Objects: Designs Against the Homeless. His edited and co-edited books include Postphenomenological Investigations, Philosophy of Science: 5 Questions, and Postphenomenology and Imaging: How to Read Technology. 